\title{
ECO-CONECTIVISMO: MODELANDO EL CONOCIMIENTO CONECTIVO
}

Diego Mosquera Uzcátegui y José Lisandro Aguilar

(pp.158-185)

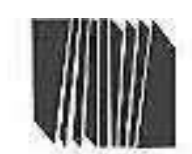

\section{Revista}

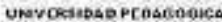

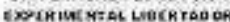

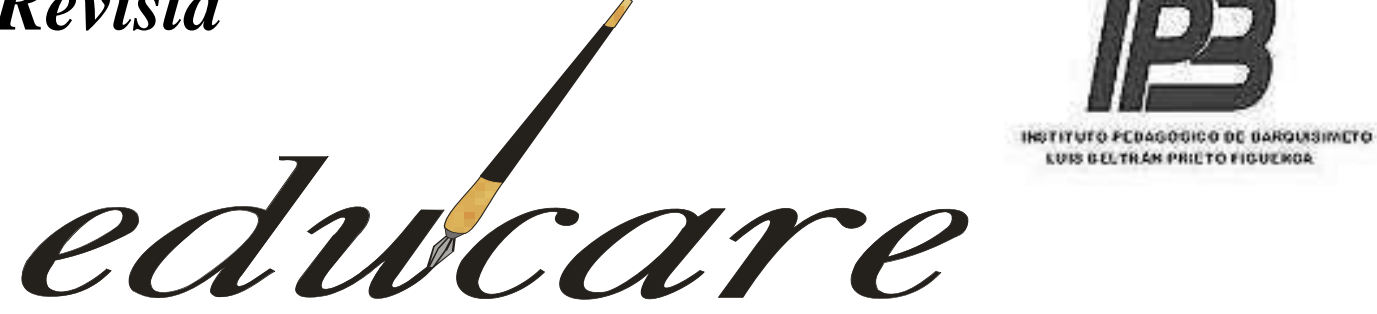

Órgano Divulgativo de la Subdirección de Investigación y Postgrado

del Instituto Pedagógico de Barquisimeto “Luis Beltrán Prieto

Figueroa"

Barquisimeto Estado Lara

Venezuela

\section{Volumen 23 № 3 Septiembre-Diciembre 2019}

ECO-CONECTIVISMO: MODELANDO EL CONOCIMIENTO CONECTIVO

ECO-CONNECTIVISM: MODELING CONNECTIVE KNOWLEDGE

\section{S S N : $2244-7296$}

\author{
Autor \\ Diego Mosquera Uzcátegui \\ José Lisandro Aguilar \\ Universidad Experimental de Guayana (UNEG) \\ Universidad de los Andes (ULA) \\ Venezuela
}

Volumen 23, $N^{\circ}$ 3, Septiembre-Diciembre 2019. ISSN 2244-7296 


\section{ECO-CONECTIVISMO: MODELANDO EL CONOCIMIENTO CONECTIVO}

Diego Mosquera Uzcátegui y José Lisandro Aguilar

(pp.158-185)

\section{Propuesta Educativa}

Recibido: 19-06-2018

\section{RESUMEN}

Este artículo presenta una propuesta educativa llamada eco-conectivismo, la cual es una forma de abordar el paradigma pedagógico conectivista en el aula de clases. Iniciamos con una discusión acerca de la influencia de las tecnologías y el aprendizaje informal en la educación formal. A partir de allí, exploramos las limitaciones de las teorías clásicas de aprendizaje para explicar el conocimiento generado en contextos de aprendizaje no estructurados. Abordamos el paradigma conectivista como estrategia para manejar estas limitaciones teóricas, y establecemos las bases de un modelo ontológico que permita su reconocimiento. Asimismo, usamos el paradigma de ecologías de conocimiento, para caracterizar un modelo de analítica de datos que permite desplegar el paradigma conectivista a partir de las interacciones que emergen en un proceso de aprendizaje. Finalmente, explicamos los detalles de su usopara un caso de estudio llamado SaCI, que es un ambiente inteligente de aprendizaje.

Descriptores: Conectivismo; paradigma conectivista; Analítica social de datos; ecologías de conocimiento.
*Diego Mosquera Aguilar **José Lisandro Aguilar Aceptado: 19-10-2019

\section{ABSTRACT}

This article presents an educational proposal called eco-connectivism, which is a way to address the connected pedagogical paradigm in the classroom. We begin with a discussion about the influence of technologies and informal learning on formal education. From there, we explorethe limitations of classical learning theories to explain the knowledge generated in unstructured learning contexts. We approach the connectivist paradigm as a strategy to manage these theoretical limitations, and lay the foundations for an ontological model that allows their recognition. We also use the paradigm of knowledge ecologies, to characterize a data analytics model that allows the connective paradigm to be deployed from the interactions that emerge in a learning process. Finally, we explain the details of its usefor a case study called $\mathrm{SaCI}$, which is an intelligent learning environment

Keywords:Connectivism; connectivist paradigm; Social data analytics; ecologies of knowledge.

* CITEC, Universidad Experimental de Guayana, Puerto Ordaz, Venezuela. INTEC, Universidad Argentina de la Empresa, Buenos Aires, Argentina Ingeniero de Sistemas, Magister en Modelado y Simulación de Sistemas, candidato a Dr. en Ciencias de la Ingeniería. Profesor Universitario e Investigador en el área de Inteligencia Artificial Distribuida. Correo electrónico: dmosquera@gmail.com

** CEMISID, Facultad de Ingeniería, Universidad de Los Andes, Mérida, Venezuela. Departamento de Informática y Sistemas, Universidad EAFIT, Medellín, Colombia. Ingeniero de Sistemas, Universidad de los Andes (ULA), 1987; Magister en Informatica, Universite Paul Sabatier, Francia,1991; Doctor en Ciencias Computacionales, Universidad Rene Descartes-Paris, France, 1995, Postdoctorados en la Universidad de Houston, 2001, y en el Laboratorie d'Automatique et Analyses de Systemes-CNRS, Toulouse-France, 2012. Profesor Titular Jubilado del Departamento de Computación de la Universidad de los Andes (ULA). Correo electrónico: aguilar@ula.ve

Volumen 23, N³, Septiembre-Diciembre 2019. ISSN 2244-7296 


\section{ECO-CONECTIVISMO: MODELANDO EL CONOCIMIENTO CONECTIVO \\ Diego Mosquera Uzcátegui y José Lisandro Aguilar}

$(p p .158-185)$

\section{Introducción}

La educación formal se ha visto fuertemente influenciada por paradigmas emergentes de aprendizaje, que han sido fruto del desarrollo de las Tecnologías de Información y Comunicación (TIC). En particular, los avances en la Web han proporcionado espacios de esparcimiento del conocimiento, posibilitando procesos de aprendizaje guiados por intereses particulares. A este modo de adquirir conocimiento, algunos autores lo han denominado aprendizaje informal (Cobo \& Moravec, 2011).

El aprendizaje informal se caracteriza por ser un aprendizaje continuo y espontáneo, llevado a cabo fuera del contexto de la educación formal. Es un hecho social en el que el individuo interactúa con todo el entorno que lo rodea, de una manera no determinada y no intencional. Es un complemento al aprendizaje formal, por cuanto refuerza el conocimiento con aptitudes, tanto personales como sociales. En el aprendizaje informal, las actividades de aprendizaje emergen naturalmente de acuerdo a las preferencias y estilo de aprender de cada individuo. Es un proceso auto-gestionado, que no recibe una instrucción ni evaluación para el abordaje, donde la calidad del conocimiento puede ser medida por el impacto que produce en el entorno social donde se desenvuelve.

Los espacios donde tiene lugar hoy en día el aprendizaje informal es proporcionado, principalmente, por las TIC; especialmente, aquellas que funcionan a través de Internet (Ibañez, 2003). Un ejemplo de esos espacios son las Redes Colaborativas de Aprendizaje. Dichos espacios han permitido desarrollar en los individuos competencias transmedia; una serie de habilidades relacionadas con la producción, el intercambio y el consumo de conocimiento usando medios interactivos digitales. Por ello, han surgido los paradigmas emergentes de aprendizaje, como una forma de responder a las limitaciones de las teorías clásicas de aprendizaje (como el constructivismo, el conductismo y el cognitivismo).

Los paradigmas emergentes de aprendizaje buscan proporcionar mecanismos para mediar con la no linealidad y la incertidumbre del entorno. Los paradigmas emergentes de

Volumen 23, N³, Septiembre-Diciembre 2019. ISSN 2244-7296 


\section{ECO-CONECTIVISMO: MODELANDO EL CONOCIMIENTO CONECTIVO}

Diego Mosquera Uzcátegui y José Lisandro Aguilar

(pp.158-185)

aprendizaje surgen por las dinámicas nuevas introducidas por las TIC, lo cual implica una necesaria transformación de las formas de adquirir y almacenar el conocimiento. En específico, los paradigmas emergentes de aprendizaje suponen un mundo visto como un cerebro social emergente: una interconexión híbrida de individuos y programas (software), en donde los individuos son las neuronas en la red, conectadas por la sinapsis producida por el software social.

En particular, el conectivismo (Siemens, 2005) es uno de esos paradigmas emergentes de aprendizaje, que se ha propuesto como una alternativa para los contextos educativos cuyas conductas de aprendizaje son impredecibles. Él no sustituye a las teorías clásicas de aprendizaje, sino que las complementa. Por ejemplo, el conectivismo puede incorporarse a un esquema de aprendizaje aprender-haciendo (un paradigma constructivista), utilizando un tipo de aprendizaje inmersivo (e.g. juegos serios colaborativos), lo que conlleva a un entorno de aprendizaje interactivo abierto y auto-gestionado.

En este artículo, presentamos el eco-conectivismo; un marco de referencia para implementar entornos conectivistas de aprendizaje. Este concepto describe las reglas que permiten la creación y gestión de ecologías del conocimiento basadas en dominios, y define un espacio para formar, establecer, analizar y evaluar las redes que emergen como consecuencia del aprendizaje. Nuestra propuesta se enfoca en explorar y explotar las capacidades meta-cognitivas de los aprendices, en espacios que combinan el aprendizaje formal con un tipo de aprendizaje auto-organizado (informal), para conformar el ecoconectivismo. Como caso de estudio, planteamos el uso del paradigma de aprendizaje constructivista aprender haciendo en el contexto de un salón inteligente, e instanciamos sus propiedades inmersivas de aprendizaje usando los principios del eco-conectivismo. Cada aprendiz del modelo es representado por su Entorno Personal de Aprendizaje (PLE, por sus siglas en inglés Personal Learning Environment). Para llevar a cabo el análisis y la interpretación del conocimiento generado, medimos el desempeño de las Redes Personales de

Volumen 23, $N^{\circ} 3$, Septiembre-Diciembre 2019. ISSN 2244-7296 


\section{ECO-CONECTIVISMO: MODELANDO EL CONOCIMIENTO CONECTIVO}

Diego Mosquera Uzcátegui y José Lisandro Aguilar

(pp.158-185)

Conocimiento (PKN, por sus siglas en inglés Personal Knowledge Network), usando técnicas de analítica de datos y analítica social de datos.

\section{Argumentación Teórica}

\section{Aspectos filosóficos del Conectivismo}

\section{Contextualizaciones Básicas}

Los avances de la ciencia en la comprensión del funcionamiento del cerebro y la influencia de Internet en ámbitos como la educación, han permitido nuevas formas de explicar cómo aprenden los seres humanos. El conectivismo es una propuesta que trata de exponer el aprendizaje en la era digital. En la Tabla, se exponen los temas que aborda el aprendizaje conectivista

\section{Tabla 1}

El conectivismo y los temas de aprendizaje que debe abordar

\begin{tabular}{|c|c|}
\hline Cuestión & Conectivismo \\
\hline $\begin{array}{l}\text { ¿Cómo ocurre el } \\
\text { aprendizaje? }\end{array}$ & $\begin{array}{l}\text { Distribuido dentro de una red, social, extendida tecnológicamente, } \\
\text { reconociendo o interpretando patrones. }\end{array}$ \\
\hline $\begin{array}{l}\text { ¿Qué papel desempeña la } \\
\text { memoria? }\end{array}$ & $\begin{array}{l}\text { Dos funciones básicas: 1) almacenar la información y modelos } \\
\text { conceptuales necesarios para el reconocimiento y la localización; y 2) } \\
\text { fortalecer las conexiones del proceso de aprendizaje. }\end{array}$ \\
\hline $\begin{array}{l}\text { ¿Cuál es el papel de la } \\
\text { motivación? }\end{array}$ & $\begin{array}{l}\text { Motivación Intrínseca, ya que el aprendizaje es autónomo. Esto se } \\
\text { traduce en un óptimo desarrollo de PLE. }\end{array}$ \\
\hline $\begin{array}{l}\text { ¿Cómo ocurre la } \\
\text { transferencia? }\end{array}$ & Conectando (agregando) redes. \\
\hline $\begin{array}{l}\text { ¿Qué procesos participan en } \\
\text { la auto-regulación? }\end{array}$ & $\begin{array}{l}\text { La metacognición, la auto-organización (formación espontánea de } \\
\text { estructuras, patrones o comportamientos bien organizados, a partirde } \\
\text { condiciones iniciales aleatorias), la motivación. }\end{array}$ \\
\hline ¿Qué factores influyen? & Diversidad en la red, la fuerza de los vínculos. \\
\hline
\end{tabular}

Fuente: (Siemens, Knowing knowledge, 2006).

Esta propuesta emerge como resultado del análisis de las teorías clásicas de aprendizaje en relación con las TIC (Siemens, 2005). El conectivismo combina el constructivismo y el cognitivismo, para explicar el aprendizaje complejo en un mundo social digital, diverso y no

Volumen 23, $N^{\circ} 3$, Septiembre-Diciembre 2019. ISSN 2244-7296 


\section{ECO-CONECTIVISMO: MODELANDO EL CONOCIMIENTO CONECTIVO}

Diego Mosquera Uzcátegui y José Lisandro Aguilar

(pp.158-185)

estructurado, en rápida evolución. Además, aborda el aprendizaje usando principios de teorías matemáticas (caos, complejidad y redes), para proponer un modelo de autoorganización cognitivo-social, cuyas leyes y condiciones iniciales sean causadas por necesidades de aprendizaje.

Esta teoría tiene su fundamento epistemológico en el conocimiento conectivo, referido al conocimiento creado por medio de interacciones entre entidades conectadas. De acuerdo con (Siemens, 2006), “...una propiedad de una entidad debe conducir a, o convertirse en, una propiedad de otra entidad para que puedan ser consideradas como conectadas; el conocimiento que resulta de tales conexiones es el conocimiento conectivo...". Por tanto, el conocimiento conectivo es algo más que una simple relación, es el conocimiento implícito derivado de las interacciones. En el conocimiento conectivo, el significado es un fenómeno emergente que surge de las conexiones entre entidades subyacentes.

El principio del conocimiento conectivo es muy simple: "si una mente humana puede llegar a saber, y si la mente humana es, en esencia, una red, entonces, cualquier red puede llegar a saber". En la mente, las entidades subyacentes que producen significados son las neuronas y las zonas neuronales con roles específicos. En una sociedad, las entidades subyacentes son los individuos y sus redes sociales (Siemens, 2006).

Se puede ver que en el Conectivismo, el conocimiento es social. Es una propiedad de la sociedad trabajando como un todo (sabiduría de las multitudes), formada por las conexiones entre sus miembros individuales (Aguilar, 2014). El conectivismo reconoce la existencia de la diversidad, por lo que la organización de las redes admite la "libre escala": algunas entidades de la red tienen mayor grado de conexión que otras. Por tanto, el proceso de formación de redes sigue un proceso dinámico, donde la presencia de una entidad lleva a otras a conectarse a ella. Cabe destacar que en una red libre de escala, cada pieza de información puede llegar a toda la red de una manera muy rápida (Barabási \& Albert, 1999).

Volumen 23, N³, Septiembre-Diciembre 2019. ISSN 2244-7296 


\section{ECO-CONECTIVISMO: MODELANDO EL CONOCIMIENTO CONECTIVO}

Diego Mosquera Uzcátegui y José Lisandro Aguilar

(pp.158-185)

\section{Saber, Aprendizaje, Verdad y Conocimiento}

En las teorías clásicas de aprendizaje, "saber" algo significa obtener conocimiento sobre un objeto. Este conocimiento puede ser cualitativo, definido como aquel que obtiene el individuo a través de sus sentidos; o cuantitativo, definido como aquel que obtiene el individuo a través del razonamiento (Dawnes, 2012). Estos dos tipos de conocimiento explican la mayor parte de lo que se sabe de las cosas que existen en el mundo. Estos conocimientos combinan lo mejor de las capacidades humanas: percibir y razonar. Constituyen la base del lenguaje, el fundamento de la lógica y el fundamento de todas las ciencias de hoy.

Sin embargo, a principios del siglo XXI, se hizo evidente un tercer tipo de conocimiento. Uno que existe por encima de aquellos derivados de los sentidos y el razonamiento (Dawnes, 2012). Nos referimos al conocimiento conectivo, aquel basado en las conexiones que existen en el mundo y obtenido desde esas conexiones. Este tercer tipo de conocimiento no es independiente de los dos primeros (cualitativo y cuantitativo). El conectivismo es un paradigma que lo describe, en el que "saber" algo es estar organizado de cierta manera (exhibir patrones de conectividad). Por tanto, aprender significa adquirir ciertos patrones topológicos. En cuanto a la verdad, se establece el requisito de evidencia total: si todas las entidades involucradas (o la mayoría) concluyen el mismo hecho acerca de un fenómeno, entonces el hecho concluido tiene validez en la sociedad (consenso social) y, por tanto, es considerado "verdad".

En cuanto a la existencia de conocimiento, es importante llevar a cabo un análisis cualitativo de las redes. De acuerdo a lo que plantea el conectivismo, las redes de conocimiento deben cumplir con las siguientes propiedades (Downes, 2007): diversidad, autonomía, interactividad y apertura, tal como se muestra en la 2.

Volumen 23, $N^{\circ} 3$, Septiembre-Diciembre 2019. ISSN 2244-7296 


\section{ECO-CONECTIVISMO: MODELANDO EL CONOCIMIENTO CONECTIVO}

Diego Mosquera Uzcátegui y José Lisandro Aguilar

(pp.158-185)

\section{Tabla 2}

Condiciones para una red de conocimiento conectivo

\begin{tabular}{|c|c|c|}
\hline Propiedades & Temas relacionados & Abordaje \\
\hline Autonomía & $\begin{array}{l}\text { ¿Los conocedores individuales contribuyen } \\
\text { a la interacción por su propia voluntad, de } \\
\text { acuerdo a sus propios conocimientos y } \\
\text { valores?, o ¿actúan a instancias de un agente } \\
\text { externo, buscando amplificar un cierto punto } \\
\text { de vista, a través de la cantidad, antes que la } \\
\text { razón y la reflexión? }\end{array}$ & $\begin{array}{l}\text { Cada participante tiene poder de decisión } \\
\text { sobre el nivel de desarrollo, énfasis y } \\
\text { objetivos específicos de aprendizaje, así } \\
\text { como en el monitoreo y evaluación de los } \\
\text { mismos Su participación responde a un } \\
\text { interés personal, autónomo, y refleja una } \\
\text { posición personal en los temas y actividades } \\
\text { desarrollados. }\end{array}$ \\
\hline Interactividad & $\begin{array}{l}\text { ¿El conocimiento que se genera es producto } \\
\text { de una interacción entre sus miembros, o es } \\
\text { una agregación simple de las perspectivas de } \\
\text { los miembros? }\end{array}$ & $\begin{array}{l}\text { Los procesos de aprendizaje y construcción } \\
\text { se producen en la interacción con otras } \\
\text { personas, medios y recursos. }\end{array}$ \\
\hline Diversidad & $\begin{array}{l}\text { ¿El proceso de aprendizaje involucra el } \\
\text { mayor espectro posible de puntos de vista?, } \\
\text { ¿las personas que interpretan el tema de una } \\
\text { manera y desde un conjunto de supuestos, } \\
\text { interactuaron con quienes se acercan al tema } \\
\text { desde perspectivas diferentes? }\end{array}$ & $\begin{array}{l}\text { La diversidad de enfoques, fuentes y } \\
\text { modelos se articula con el reconocimiento } \\
\text { de la diversidad de los individuos, abriendo } \\
\text { espacios que permitan la exploración de } \\
\text { intereses específicos. }\end{array}$ \\
\hline Apertura & $\begin{array}{l}\text { ¿Existe un mecanismo que le permita a un } \\
\text { punto de vista dado entrar en el sistema, ser } \\
\text { escuchado e interactuar con los demás? }\end{array}$ & $\begin{array}{l}\text { Tanto en el acceso a contenidos como a } \\
\text { actividades, se busca la inclusión de un } \\
\text { espectro amplio de puntos de vista que } \\
\text { enriquezcan la perspectiva de los } \\
\text { individuos. Esto lleva al reconocimiento de } \\
\text { que no hay una única respuesta correcta. }\end{array}$ \\
\hline
\end{tabular}

Fuente: Basado en el trabajo de Stephen Downes (2007).

Las cuatro propiedades mostradas en la Tabla, se denominan de aquí en adelante AIDA, describen los parámetros que influencian un proceso de aprendizaje emergente. En particular, los patrones topológicos que surgen durante un proceso de aprendizaje conectivista, pueden ser verificados en términos de AIDA, como una forma para la cualificación del conocimiento conectivo.

Por otra parte, el Conectivismo presenta una visión en etapas de cómo los individuos en red exploran y encuentran conocimientos de una manera ecológica, como se muestra en la siguiente tabla:

Volumen 23, $N^{\circ} 3$, Septiembre-Diciembre 2019. ISSN 2244-7296 


\section{ECO-CONECTIVISMO: MODELANDO EL CONOCIMIENTO CONECTIVO}

Diego Mosquera Uzcátegui y José Lisandro Aguilar

(pp.158-185)

\section{Tabla 3}

Etapas del Conectivismo

\begin{tabular}{|c|c|}
\hline ETAPA & DESCRIPCIÓN \\
\hline $\begin{array}{l}\text { Conciencia y } \\
\text { receptividad }\end{array}$ & $\begin{array}{l}\text { los individuos adquieren competencias básicas para el manejo de la abundancia de } \\
\text { información; tienen acceso a recursos y herramientas }\end{array}$ \\
\hline $\begin{array}{l}\text { Formación de } \\
\text { conexiones }\end{array}$ & $\begin{array}{l}\text { los individuos utilizan las competencias adquiridas en la primera etapa, para crear y } \\
\text { formar la PKN. En este nivel, los individuos están activos en el espacio de la ecología del } \\
\text { conocimiento, en términos de consumir o adquirir nuevos recursos o herramientas. }\end{array}$ \\
\hline $\begin{array}{l}\text { Contribución e } \\
\text { implicación }\end{array}$ & $\begin{array}{l}\text { los individuos comienzan a contribuir activamente en la ecología de conocimiento, } \\
\text { transformándose en un "nodo visible". }\end{array}$ \\
\hline $\begin{array}{l}\text { Reconocimiento } \\
\text { de patrones }\end{array}$ & $\begin{array}{l}\text { los individuos son conocedores competentes de la ecología del conocimiento y, como } \\
\text { participantes dinámicos, han pasado del consumo pasivo de contenido a la contribución } \\
\text { activa. En este nivel, los individuos son capaces de reconocer las propiedades eficientes de } \\
\text { las redes de conocimiento (e.g. desempeño, capacidad de colaboración, experticia). }\end{array}$ \\
\hline $\begin{array}{l}\text { Creación de } \\
\text { significado }\end{array}$ & $\begin{array}{l}\text { los individuos son capaces de comprender el significado como fundamento de la acción, } \\
\text { lo que le permite reformar puntos de vista, perspectivas y opiniones. }\end{array}$ \\
\hline Praxis & $\begin{array}{l}\text { los individuos son capaces de efectuar el proceso cíclico de la reflexión, experimentación } \\
\text { y acción, para evaluar de una manera crítica las herramientas, procesos y elementos de } \\
\text { una ecología de conocimiento. }\end{array}$ \\
\hline
\end{tabular}

Vale destacar, que en este tipo de conocimiento conectivo, existen un conjunto de herramientas, fuentes de información, conexiones y actividades que cada individuo utiliza de forma asidua para aprender denominado Entorno Personal de Aprendizaje (PLE), el cual se configura por los procesos, experiencias y estrategias, que el aprendiz puede poner en marcha para aprender, el cual está determinado por las posibilidades que las tecnologías abren y potencian.

Un PLE, se encuentra constituido por herramientas (h), mecanismos (m) y actividades (a) para: leer, que son las fuentes documentales de información (ej: blogs y wikis), para hacer/reflexionar haciendo (ej: canales de video) y para compartir/reflexionar en comunidad (ej: redes sociales). Asimismo, requiere de criterios de decisión en el ámbito del aprendizaje formal como la orientación al currículo, las fuentes de información, selección de las herramientas, estrategias de aprendizaje formal, colaboración, socialización, valores y ética en el uso de la web y seguridad.

Volumen 23, $N^{\circ} 3$, Septiembre-Diciembre 2019. ISSN 2244-7296 


\section{ECO-CONECTIVISMO: MODELANDO EL CONOCIMIENTO CONECTIVO}

Diego Mosquera Uzcátegui y José Lisandro Aguilar

$(p p .158-185)$

\section{Descripción de la Propuesta}

En esta sección, describimos las bases de nuestro modelo pedagógico de gestión, llamado eco-conectivismo. Particularmente, diseñamos un marco de referencia para implementar el conectivismo, en un entorno de aprendizaje ubicuo (que se produce en cualquier lugar y momento) gestionado por tecnologías computacionales. Las bases de nuestra propuesta se inspiran en conceptos provenientes de la ecología (razón del prefijo eco), la optimización, la analítica de datos, los sistemas inteligentes, y la computación autonómica.

\section{Eco-Conectivismo}

Como hemos mencionado, el conectivismo surge como una manera de responder a las limitaciones que tienen las teorías clásicas de aprendizaje, en cuanto a cuatro aspectos fundamentales: 1) el lugar donde ocurre el aprendizaje; 2) la dificultad de adaptarse a entornos no estructurados o contextos imprecisos; 3) la necesidad de controlar y predecir el comportamiento del aprendiz; y 4) la imposibilidad de lidiar con el enorme flujo de información y la obsolescencia del conocimiento. En otras palabras, el conectivismo busca complementar el conocimiento cuantitativo y cualitativo centrado en el individuo, con un nivel de conocimiento conectivo, centrado en las redes de individuos que emergen en una ecología del conocimiento.

Sin embargo, el conectivismo también tiene sus propias limitaciones. Como se explica en (García, 2009), estas limitaciones se relacionan con tres aspectos fundamentales:

1. Información y conocimiento: por la diversidad de nodos y conocimiento a ser explorados, es difícil garantizar requisitos de disponibilidad, calidad y correctitud; necesarios para formar conexiones que garanticen el aprendizaje.

2. Enseñanza y aprendizaje: el conocimiento conectivo requiere un cambio de paradigma en cuanto a la metodología de enseñanza y aprendizaje. En lugar de que la actividad educativa se centre en el diseño de cursos, es necesario dirigir los esfuerzos en el diseño de

Volumen 23, $N^{\circ} 3$, Septiembre-Diciembre 2019. ISSN 2244-7296 


\section{ECO-CONECTIVISMO: MODELANDO EL CONOCIMIENTO CONECTIVO}

Diego Mosquera Uzcátegui y José Lisandro Aguilar

(pp.158-185)

PLE y ecologías del conocimiento, para que los individuos busquen y establezcan sus PKN, basado en sus intereses y necesidades.

3. Formalidad y capacitación: las instituciones educativas deben capacitarse con los recursos tecnológicos necesarios para integrar procesos emergentes de aprendizaje a la educación formal. Esto último incluye, modelos de gestión que permitan captar y valorar el proceso de aprendizaje en todos y cada uno de sus niveles, y el de conocimiento generado.

En este orden de ideas, proponemos un marco tecnológico referencial basado en modelos de analítica de datos, que permita establecer y gestionar ecologías del conocimiento; respondiendo a los requisitos de disponibilidad, calidad y correctitud, tanto de los recursos como de las PKN, necesarios para garantizar el aprendizaje conectivo. Además, nuestro marco de referencia define las bases conceptuales y tecnológicas para diseñar, mantener y gestionar PLE, en entornos educativos formales, informales, o combinaciones de ambos.

\section{Bases del Eco-Conectivismo}

Para el conectivismo, el aprendizaje es concebido como un "organismo vivo", cuyo proceso de formación es no estructurado e impredecible (Siemens, 2005). De acuerdo a las leyes naturales de la ecología, un organismo vivo es un elemento de alta complejidad, con propiedades de auto-adaptación, que nace en un entorno, crece, alcanza la capacidad para reproducirse, y muere. Estos organismos tienen una estructura interna definida, son un sistema dotado de organización, en constante relación con el entorno. Este entorno donde se produce el ciclo de vida de los organismos se denomina ecosistema, el cual también evoluciona de manera natural, de acuerdo a su dinámica interna. A este proceso de evolución se le conoce como sucesión ecológica; término que alude al aspecto esencial de sustitución de especies dentro de un ecosistema.

La sucesión ecológica es el proceso de cambio (fisiológico, morfológico y de comportamiento), que rige la evolución de los organismos en un ecosistema, y que podemos observar tanto en el tiempo como en el espacio. Supone la sustitución de unas comunidades

Volumen 23, $N^{\circ} 3$, Septiembre-Diciembre 2019. ISSN 2244-7296 


\section{ECO-CONECTIVISMO: MODELANDO EL CONOCIMIENTO CONECTIVO}

Diego Mosquera Uzcátegui y José Lisandro Aguilar

(pp.158-185)

de especies por otras, de manera que con el transcurso del tiempo el ecosistema se va volviendo más complejo, aumentando los tipos de interacciones y la diversidad de organismos. Igualmente, la sucesión ecológica es un proceso ordenado de auto-organización de un sistema complejo (ecosistema), que se da en una serie de etapas. Estas etapas se pueden categorizar en tres: 1) etapas iniciales o de constitución, dominadas por especies de las que en el lenguaje ecológico y evolutivo se llaman pioneras, oportunistas, desde el punto de vista de sus requerimientos de recursos, con una estrategia reproductiva basada en la producción de muchos descendientes; 2) etapas intermedias o de maduración, establecidas por especies más complejas, capaces de aportar recursos al ecosistema; y 3) etapas finales o "clímax", caracterizadas por especies especialistas, en cuanto al uso de recursos, con baja tasa de reproducción.

En este orden de ideas, definimos el eco-conectivismo, como un modelo pedagógico conectivista y referencial, inspirado en conceptos provenientes de la ecología, cuyo objetivo es observar y guiar la evolución del aprendizaje en ecologías del conocimiento. En particular, utilizamos el concepto de sucesión ecológica para describir, de manera precisa, los momentos pedagógicos del aprendizaje conectivista. Como el aprendizaje conectivista se manifiesta en las conexiones que forman las PKN, y estas redes son formadas por individuo y sus interacciones con el conjunto de recursos y herramientas tecnológicas de consulta, reflexión, experimentación, interacción y acción; entonces, para el eco-conectivismo, los organismos vivos a observar son los PLE; y el ambiente a gestionar (sucesión ecológica) son agrupaciones de PLE, denominadas ecosistemas de aprendizaje.

\section{Sucesión Ecológica en el Eco-Conectivismo}

El eco-conectivismo tiene dos objetivos fundamentales: 1) crear las bases referenciales para definir entornos de aprendizaje conectivistas. Esto es, abordar, gestionar y evaluar las etapas del conectivismo, con mecanismos precisos para abordar sus limitaciones; y 2) modelar el conocimiento conectivo. Esto es, definir las estrategias necesarias para garantizar

Volumen 23, N³, Septiembre-Diciembre 2019. ISSN 2244-7296 


\section{ECO-CONECTIVISMO: MODELANDO EL CONOCIMIENTO CONECTIVO}

Diego Mosquera Uzcátegui y José Lisandro Aguilar

(pp.158-185)

que la dinámica evolutiva del proceso de aprendizaje en las ecologías del conocimiento cumpla con las condiciones de AIDA como propiedades emergentes, cruciales para el ecoconectivismo.

Para lograr estos objetivos, se definen tres procesos evolutivos del aprendizaje, los cuales relacionamos con las etapas iniciales, intermedias y finales de un modelo de sucesión ecológica, correspondiendo éstas a los momentos pedagógicos de un proceso de aprendizaje conectivista. La figura 1 muestra los procesos del eco-conectivismo.

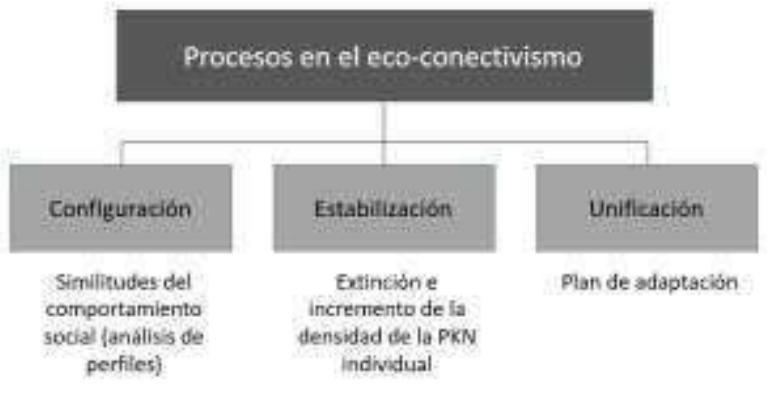

Figura 1. Procesos del eco-conectivismo

En el proceso de configuración, el eco-conectivismo propone la evaluación de la dinámica de interacción aprendiz-entorno para generar ecosistemas homogéneos de PLE, en base al análisis de la similitud del comportamiento social. En el proceso de estabilización, el ecoconectivismo propone una partición de la ecología en grupos de ecosistemas aptos y noaptos, basados en la calidad de las aportaciones de los individuos y las propiedades de AIDA adquiridas. En el proceso de unificación, el eco-conectivismo distribuye los individuos presentes en ecosistemas no-aptos a los ecosistemas aptos, en base a un análisis de distancia y similitud, enriquecido con un plan de adaptación que facilita la inserción y supervivencia de los individuos en el nuevo ecosistema.

En un proceso de aprendizaje eco-conectivista, las fases aparecen de forma secuencial y cíclica, y actúan de manera paralela, sobre los dos elementos esenciales de la ecología del conocimiento: el PLE y los ecosistemas de aprendizaje. El proceso evolutivo de los PLE se

Volumen 23, N³, Septiembre-Diciembre 2019. ISSN 2244-7296 


\section{ECO-CONECTIVISMO: MODELANDO EL CONOCIMIENTO CONECTIVO}

Diego Mosquera Uzcátegui y José Lisandro Aguilar

(pp.158-185)

enmarca en las competencias a lograr por las etapas del conectivismo (conciencia y receptividad, formación de conexiones, contribución e implicación, reconocimiento de patrones, creación de significado y praxis). En la medida que el proceso de aprendizaje avanza, se incorporan, de manera selectiva y personalizada, recursos de aprendizaje y herramientas tecnológicas en cada nivel estructural de los PLE, de acuerdo a la etapa actual de desarrollo del mismo. Por otro lado, el proceso evolutivo de los ecosistemas de aprendizaje, es guiado por las condiciones fundamentales del eco-conectivismo (AIDA).

Durante el proceso de aprendizaje, el eco-conectivismo busca la convergencia de estas condiciones dentro de la ecología del conocimiento. A este momento de convergencia en la etapa final de la sucesión ecológica se le conoce como "clímax", y es el punto de desarrollo de los ecosistemas en el que se dan las condiciones de equilibrio entre todos sus componentes con el máximo de diversidad de especies.

\section{Eco-Conectivismo y Entorno}

Un PLE está diseñado para construirse en base a herramientas tecnológicas que faciliten el proceso de aprendizaje conectivista, y su ambiente natural de desarrollo es la Web (Internet). Cuando un individuo interacciona en la Web, genera una cantidad de datos que quedan registrados en Internet. Estos datos están relacionados con los recursos utilizados (sitios Web, blogs o repositorios visitados), conversaciones (redes sociales, chats, foros, etc.), entre otras cosas, que pueden ser integrados en el PLE, en correspondencia con la intención de su uso (leer, reflexionar o compartir).

Por otro lado, los datos que genera un individuo en la Web pueden ser observados para cuantificar, medir e informar sobre la actividad del individuo en el entorno. De esta manera, podemos determinar los tipos de recursos de aprendizaje que un individuo particular incorpora a su PLE para leer, los medios que utiliza para reflexionar sobre lo que lee, y los individuos con los que interactúa durante su proceso de aprendizaje.

Volumen 23, N³, Septiembre-Diciembre 2019. ISSN 2244-7296 


\section{ECO-CONECTIVISMO: MODELANDO EL CONOCIMIENTO CONECTIVO}

Diego Mosquera Uzcátegui y José Lisandro Aguilar

(pp.158-185)

Las métricas relacionadas a sitios Web y social media, pueden ser utilizadas para extraer conocimiento acerca del individuo (o grupos de individuos), en relación a un proceso u objetivo organizacional. A esta forma de extraer conocimiento de Internet se le denomina "analítica de datos" (Aguilar, Riofrio, Encalada, \& Guamán, 2015; Aguilar, Cordero \& Buendia, 2018; Aguilar, Sanchez, Cordero, Valdiviezo, Barba \& Chamba, 2018).En general, existen diversos enfoques para hacer analítica de datos: analítica Web, analítica social, analítica móvil, analítica de big-data, entre otros. Cada uno de estos enfoques busca capturar, procesar e interpretar los datos generados por los individuos en Internet, desde perspectivas particulares (comportamiento en la Web, relaciones en la Web, etc.).

En ese orden de ideas, en cada proceso (configuración, estabilización y unificación) del eco-conectivismo se propone realizar una serie de análisis de datos, para determinar el comportamiento (o patrones) que surge de la interacción del individuo con el ambiente (a través del PLE). Esta interacción se da, esencialmente, en entornos colaborativos y sociales desplegados en la Web (Internet), cuyas herramientas son capaces de capturar y registrar todos los datos relacionados con la interacción individuo-entorno. Para lograr esto, es necesario identificar la tarea (o conjunto de tareas) de analítica de datos asociada(s) a cada uno de los procesos del eco-conectivismo. La combinación de estas tareas de analítica de datos permitirá, eventualmente, alcanzar los objetivos y las condiciones de estabilidad del proceso de aprendizaje eco-conectivista. En las siguientes secciones definimos estas tareas de analítica de datos.

\section{Modelo de Adaptación en el Eco-Conectivismo}

Como hemos dicho, el eco-conectivismo es un marco referencial, inspirado en conceptos provenientes de la ecología, dirigido a gestionar y optimizar ambientes de aprendizaje conectivistas, usando herramientas computacionales. En un ambiente eco-conectivista, los elementos constitutivos son ecosistemas de PLE que evolucionan en una ecología de

Volumen 23, $N^{\circ} 3$, Septiembre-Diciembre 2019. ISSN 2244-7296 


\section{ECO-CONECTIVISMO: MODELANDO EL CONOCIMIENTO CONECTIVO}

Diego Mosquera Uzcátegui y José Lisandro Aguilar

(pp.158-185)

conocimiento. Esta evolución es cíclica y continua, dirigida por los procesos de configuración, estabilización y unificación ecológica del eco-conectivismo.

Este esquema evolutivo de PLE describe un proceso iterativo de optimización basado en la adaptación recurrente del PLE en relación a los recursos incorporados. Para los dos primero niveles del PLE (leer y reflexionar), los recursos son seleccionados y asignados usando información personalizada de los individuos (e.g. estilo de aprendizaje, condiciones especiales o contextuales, etc). El objetivo de este modelo de adaptación es llegar a la configuración de un PLE relativo a la etapa de "praxis"del conectivismo (ver figura 1).Para el tercer nivel del PLE (la PKN), definimos una Función de Aptitud (FA) que busca determinar las agrupaciones de PLE basados en algún criterio de similitud. Esta tarea de agrupamiento, corresponde al primer proceso del eco-conectivismo (ver Figura 1).

En general, dentro de un ecosistema se reconocen dos tipos de conexiones entre individuos: conexiones fuertes y conexiones débiles. Las conexiones fuertes son aquellas que se dan entre individuos que pertenecen a un mismo ecosistema. Las conexiones débiles se dan entre individuos de ecosistemas diferentes. Para medir la aptitud de un ecosistema de aprendizaje, se toman en cuenta los siguientes parámetros de la ecología de conocimiento:

1. U: umbral de supervivencia ecológica,

2. D: cantidad de conexiones débiles totales de la ecología,

3. E: cantidad de conexiones débiles del ecosistema

La FA es definida como el cociente E/D. El umbral de supervivencia ecológica es un parámetro establecido para especificar el grado de diversidad deseado en la ecología. Este parámetro es un valor entre cero y uno. Mientras mayor sea el valor del umbral de supervivencia, mayor será el grado de diversidad especificado.

Cuando el cociente E/D para un ecosistema dado es menor o igual a U, entonces el ecosistema en cuestión es considerado no apto. Cuando coexisten ecosistemas aptos y no aptos dentro de la ecología, se está en presencia de una distribución ecológica inestable. La presencia de ecosistemas no aptos dentro de una ecología de conocimiento, permite la

Volumen 23, $N^{\circ} 3$, Septiembre-Diciembre 2019. ISSN 2244-7296 


\section{ECO-CONECTIVISMO: MODELANDO EL CONOCIMIENTO CONECTIVO}

Diego Mosquera Uzcátegui y José Lisandro Aguilar

(pp.158-185)

ejecución de tareas de selección de entidades inmersas en ecosistemas no aptos, para ejecutar tareas de migración de estas entidades a ecosistemas cuya FA esté sobre el umbral D. Estas tareas de selección y migración de entidades se asocian al proceso/fase de estabilización del eco-conectivismo.

Esta forma de selección y migración puede causar una inestabilidad fisiológica de las entidades (PLE) y ecosistemas involucrados. Esto requiere de la ejecución de un plan de adaptación que permita disminuir los efectos que puede causar el proceso de migración. La ejecución de este plan se asocia a tareas de recomendación de recursos de aprendizaje, como forma de unificación del eco-conectivismo. El objetivo de esta heurística de optimización es responder con una ecología de conocimiento compuesta exclusivamente de ecosistemas de aprendizaje aptos, y garantizar que el proceso de aprendizaje adquiera un comportamiento coherente en relación a la AIDA.

\section{Tareas de Analítica de Datos Asociadas al Eco-Conectivismo}

En un proceso de aprendizaje abierto, el aprendizaje inmersivo, la interacción y el trabajo colaborativo utilizando la Web, generan grandes volúmenes de datos (big-data), que pueden ser aprovechados para encontrar una articulación coherente entre el aprendizaje formal e informal, en el marco del aprendizaje eco-conectivista.

Como se ha mencionado, el eco-conectivismo supone el uso de herramientas computacionales para la gestión y optimización de CLE; y una plataforma que soporte procesos de aprendizaje eco-conectivistas requiere de diversas tareas de analítica de datos que permitan la ejecución y consecución de los tres procesos asociados al eco-conectivismo.

\section{Tabla 4}

\begin{tabular}{ccccc}
$\begin{array}{c}\text { Relación proceso eco-conectivista } \\
\text { Proceso eco- } \\
\text { conectivista }\end{array}$ & $\begin{array}{c}\text { Propósito de la } \\
\text { tarea de AdD }\end{array}$ & $\begin{array}{c}\text { Técnica de } \\
\text { AdD }\end{array}$ & Tarea de AdD & Resultado esperado \\
\hline & $\begin{array}{c}\text { Descripción del } \\
\text { estado actual de la }\end{array}$ & $\begin{array}{c}\text { Minería } \\
\text { Web de uso }\end{array}$ & Agrupamiento & $\begin{array}{l}\text { Ecosistemas de aprendizaje: } \\
\text { distribución ecológica } \\
\text { inestable. }\end{array}$ \\
\hline
\end{tabular}

Volumen 23, $N^{\circ} 3$, Septiembre-Diciembre 2019. ISSN 2244-7296 


\section{ECO-CONECTIVISMO: MODELANDO EL CONOCIMIENTO CONECTIVO}

Diego Mosquera Uzcátegui y José Lisandro Aguilar

(pp.158-185)

\begin{tabular}{|c|c|c|c|c|}
\hline Configuración & $\begin{array}{l}\text { ecología del } \\
\text { conocimiento. }\end{array}$ & $\begin{array}{l}\text { Minería de } \\
\text { texto }\end{array}$ & $\begin{array}{l}\text { Enriquecimiento } \\
\text { semántico }\end{array}$ & $\begin{array}{l}\text { Ecosistemas de aprendizaje } \\
\text { con enriquecimiento } \\
\text { semántico. }\end{array}$ \\
\hline & & & Segmentación & Partición de la ecología \\
\hline Estabilización & $\begin{array}{l}\text { Predicción de lo } \\
\text { desconocido del } \\
\text { proceso de } \\
\text { aprendizaje. }\end{array}$ & $\begin{array}{l}\text { Minería de } \\
\text { grafos }\end{array}$ & Alineación & $\begin{array}{l}\text { Distribución ecológica } \\
\text { estable. }\end{array}$ \\
\hline Unificación & $\begin{array}{l}\text { Prescripción para la } \\
\text { intercesión del } \\
\text { modelo sobre la } \\
\text { ecología del } \\
\text { conocimiento. }\end{array}$ & $\begin{array}{l}\text { Filtrado } \\
\text { colaborativo }\end{array}$ & Recomendación & $\begin{array}{l}\text { Ecología del conocimiento } \\
\text { adaptada. }\end{array}$ \\
\hline
\end{tabular}

Fuente: Elaboración propia

La ¡Error! No se encuentra el origen de la referencia. muestra la relación entre los procesos eco-conectivistas y lastécnicas de analítica de datos. El proceso de configuración eco-conectivista corresponde a la etapa de constitución de la sucesión ecológica. Para lograr la constitución, el eco-conectivismo utiliza la minería de Web de uso para implementar tareas de analítica de datos que permitan descubrir ecosistemas de aprendizaje a partir del entorno social donde se desenvuelve. En específico, el proceso de configuración eco-conectivista se basa en un algoritmo de agrupamiento con enriquecimiento semántico en las interacciones, para describir un tipo de organización espontánea de grupos de PLE durante un proceso de aprendizaje.

Este agrupamiento describe una partición temporal de la ecología de conocimiento, que surge de las interacciones entre los individuos que participan en el proceso de aprendizaje. Esta partición no es necesariamente estable en cuanto a las competencias de colaboración deseadas (maduración) en el proceso de aprendizaje. Esto conlleva a realizar un análisis más detallado de la ecología en relación a la diversidad esperada. De esta manera, se propone el siguiente proceso de estabilización.

El proceso de estabilización eco-conectivista corresponde a la etapa de maduración de la sucesión ecológica. Para la maduración, el eco-conectivismo utiliza la minería de grafos para implementar tareas de analítica de datos que permitan la 2-segmentación (ecosistemas aptos

Volumen 23, N³, Septiembre-Diciembre 2019. ISSN 2244-7296 


\section{ECO-CONECTIVISMO: MODELANDO EL CONOCIMIENTO CONECTIVO}

Diego Mosquera Uzcátegui y José Lisandro Aguilar

(pp.158-185)

y no-aptos), a partir de un parámetro denominado umbral de supervivencia ecológica (diversidad esperada). Además, se utiliza la noción de centroide para establecer medidas de similitud entre las entidades de ecosistemas no-aptos (entidades en transición) y la entidad central de los ecosistemas aptos. Esto último permite implementar la tarea de alineación del eco-conectivismo, la cual se basa en un proceso de migración guiado por similitud de las entidades involucradas en ecosistemas no-aptos. Una vez llevada a cabo la alineación, se considera una distribución ecológica estabilizada.

Sin embargo, la estabilización topológica de la ecología del conocimiento no asegura el cumplimiento del proceso de adaptación; objetivo necesario para llegar a la etapa final o "clímax" de la sucesión ecológica. En este sentido, se define el proceso de unificación. El proceso de unificación eco-conectivista corresponde a la etapa de finalización de la sucesión ecológica. En este proceso, el eco-conectivismo utiliza algoritmos de filtrado colaborativo para implementar tareas de analíticas de datos que permitan la recomendación (de recursos de aprendizaje y colaboración). En particular, se utiliza el filtrado colaborativo para generar una predicción basada en la identificación de vecinos similares a un mismo individuo, y con ello recomendar los recursos de aprendizaje que deben ser incorporados al PLE. El objetivo de este proceso es enriquecer los ecosistemas de aprendizaje con la información de los individuos migrados.

A continuación, describimos las tareas de analítica de datos mencionadas en la ¡Error! No se encuentra el origen de la referencia., en relación a los procesos ecoconectivistas:

-Agrupamiento y enriquecimiento semántico: la intención del agrupamiento es la de coleccionar PLE en comunidades no superpuestas de acuerdo a un criterio de similitud. Se dice que una red tiene estructura de comunidades, si los nodos de la red pueden ser fácilmente agrupados. En el caso particular de las comunidades no superpuestas, la red se divide naturalmente en grupos de nodos densamente conectados internamente, y con pocas conexiones entre grupos. Para lograr el agrupamiento, es necesario considerar técnicas de

Volumen 23, N³, Septiembre-Diciembre 2019. ISSN 2244-7296 


\section{ECO-CONECTIVISMO: MODELANDO EL CONOCIMIENTO CONECTIVO}

Diego Mosquera Uzcátegui y José Lisandro Aguilar

(pp.158-185)

minería de datos. Particularmente, dada la naturaleza del ambiente en el que tiene lugar el eco-conectivismo, la técnica de minería de datosa considerar debe ser capaz de particionar la ecología de conocimiento en subconjuntos densamente conectados, tomando en cuenta la semántica de las relaciones en cuento al dominio y los objetivos de aprendizaje.

-Segmentación: consiste en una partición binaria de la ecología del conocimiento, que diferencia entre ecosistemas de aprendizaje aptos y no-aptos. Cada ecosistema tiene un valor de supervivencia ecológica que se obtiene a través de la FA. Una vez obtenidos aquellos ecosistemas no-aptos, se genera una lista compuesta por aquellos PLE que pertenecen a estos ecosistemas.

-Alineación: la intención de laalineación es la asociación de la lista de PLE obtenida en el proceso de segmentación,con aquellos ecosistemas aptos que aseguren a cada PLE, integrarse al ecosistema más similar. Para ello, es importante establecer medidas de similitud entre cada PLE a migrar con las comunidades candidatas. Un PLE será alineado al ecosistema de aprendizaje con el que tenga la mayor cantidad de propiedades en común.

-Recomendación: la intención del proceso de recomendación es la creación de un plan de inserción de las entidades alineadas. La ejecución del plan de inserción supone una disminución de la distancia entre la entidad alineada y las entidades de su nuevo ecosistema. En este sentido, se requiere de un sistema que capture información del PLE alineado, la combine con información histórica del ecosistema de aprendizaje, y produzca las recomendaciones de recursos de aprendizaje necesarios que deben ser incorporados al PLE. Estas tareas de AdD garantizan ecologías del conocimiento "sanas".

\section{Resultados o Hallazgos}

\section{Caso de Estudio: Aula Inteligente SaCI}

Un aula inteligente es un tipo particular de ambiente inteligente (Droege, 1997), en el que los sistemas computacionales embebidos en el "ambiente", permiten mejorar las experiencias de aprendizaje a través de espacios educativos interactivos (Olmo, Llera, \& Arias, 1999).Un

Volumen 23, $N^{\circ} 3$, Septiembre-Diciembre 2019. ISSN 2244-7296 


\section{ECO-CONECTIVISMO: MODELANDO EL CONOCIMIENTO CONECTIVO}

Diego Mosquera Uzcátegui y José Lisandro Aguilar

(pp.158-185)

aula inteligente incorpora una serie de componentes computacionales (hardware y software) para permitir la interacción, el monitoreo, control y evaluación, del proceso de aprendizaje (Sánchez \& Campos, 2014). En específico, un aula inteligente puede incorporar escritorios interactivos (por aprendiz), pizarra interactiva, cámaras inteligentes, robots sociales, sistemas de realidad aumentada, agentes artificiales inteligentes, un centro de control y monitoreo, y un software que permita la integración de todos los dispositivos mencionados (Aguilar, Valdiviezo, Cordero, \& Sánchez, 2015).

SaCI (Salón de Clase Inteligente) es un salón inteligente cuyas actividades de enseñanzaaprendizaje se establecen bajo el paradigma constructivista aprender haciendo. Tecnológicamente, SaCI está basado en un paradigma computacional de gestión inteligente denominado Sistemas Multiagentes (SMA) (Aguilar, Valdiviezo, Cordero, \& Sánchez, 2015). $\mathrm{SaCI}$ propone dos tipos de agentes, uno para caracterizar los componentes de software y otro para definir los componentes de hardware. ElSMA de SaCI está definido en (Sánchez, Aguilar, Cordero, \& Valdiviezo, 2015), y su principal objetivo es proveer un medio inteligente capaz de guiar un proceso de aprendizaje constructivista. En particular, los agentes de SaCI son capaces de capturar información acerca del aprendiz, procesarla de acuerdo a sus metas e intenciones, y actuar en consecuencia de manera inteligente (adaptada a las necesidades, desempeño y capacidades del aprendiz).El paradigma computacional utilizado en SaCI para descubrir conocimiento, usando los datos generados en él, es la Analítica de Aprendizaje (LA, por sus siglas en inglés LearningAnalitycs)(Aguilar, Cordero \& Buendia, 2018; Aguilar, Sanchez, Cordero, Valdiviezo, Barba \& Chamba, 2018); un caso particular de analítica de datos. En (Sánchez, Aguilar, Cordero, \& Valdiviezo, 2015)se proporciona una descripción detallada de los agentes de SaCI y sus capacidades.

LA en SaCI permite definir tareas para el análisis y adaptación de dos grandes aspectos:

1. El proceso de aprendizaje, y algunas de sus tareas de LA son:

- Caracterizar el proceso de aprendizaje, en cuanto al patrón de herramientas, mecanismos y actividades adecuadas a usar.

Volumen 23, N³, Septiembre-Diciembre 2019. ISSN 2244-7296 


\section{ECO-CONECTIVISMO: MODELANDO EL CONOCIMIENTO CONECTIVO}

Diego Mosquera Uzcátegui y José Lisandro Aguilar

(pp.158-185)

- Determinar el estilo de aprendizaje ideal en el proceso de aprendizaje

2. El comportamiento de los estudiantes, y algunas de sus tareas de LA son:

- Descubrir estilos de aprendizaje: determina el estilo de aprendizaje de un estudiante, o grupo, de acuerdo al modelo de Felder-Silverman.

- Determinar cómo el desempeño del estudiante varía en relación con los patrones de aprendizaje.

- Generar conocimiento acerca del comportamiento y rendimiento de los estudiantes. Por ejemplo, su nivel de contribución en el proceso de aprendizaje, los recursos de aprendizaje utilizados, entre otros.

- Analizar el proceso de evaluación, y vincularlos con los aspectos de rendimiento estudiantil.

- Recomendar recursos y actividades, basado en el conocimiento del perfil del estudiante.

- Identificar los problemas y necesidades de los estudiantes.

Estas tareas de LA para el análisis del proceso de aprendizaje en SaCI, son llevadas a cabo de manera autonómica (Aguilar, Sanchez, Cordero, Valdiviezo, Barba \& Chamba, 2018). Para ello, se definen ciclos autonómicos de tareas de LA, los cuales integran dichas tareas, para alcanzar objetivos estratégicos en SaCI. Entre esos objetivos estratégicos de SACI, tenemos (Aguilar, Valdiviezo, Cordero, \& Sánchez, 2015): (1) definir paradigmas de aprendizaje, (2) definir recursos educativos, (3) identificar estudiantes con necesidades de atención, (4) Evitar la deserción estudiantil.

Cada ciclo autonómico describe un conjunto de tareas de LA. Por ejemplo, para los objetivos 1), 2) y 3), una tarea fundamental de LA es la clasificación de estudiantes por rendimiento. En este caso de estudio, la intención es incorporar al SaCI la noción de PLE, y proveerle una nueva categoría de tareas de LA que permitan gestionarlo. En particular, enriqueceremos el análisis del proceso de aprendizaje usando el paradigma de aprendizaje eco-conectivista. Esta nueva categoría de LA tiene dos propósitos:

Volumen 23, N³, Septiembre-Diciembre 2019. ISSN 2244-7296 


\section{ECO-CONECTIVISMO: MODELANDO EL CONOCIMIENTO CONECTIVO}

Diego Mosquera Uzcátegui y José Lisandro Aguilar

(pp.158-185)

1) Extender los ciclos autonómicos de tareas del SaCI, a través de la generación de indicadores conectivistas (relacionados con cada etapa del conectivismo), para la evaluación del proceso de aprendizaje y el rendimiento estudiantil. En la tabla 5 se describen las nuevas tareas de LA para el SaCI basadas en el paradigma eco-conectivista. Esto enriquece de información a los ciclos autonómicos de tareas del SaCI, y por ende mejora la toma de decisiones.

2) Caracterizar un nuevo ciclo autonómico de tareas para coadyuvar a procesos de aprendizaje conectivista. Esto es, realizar intervención autonómica sobre el proceso de aprendizaje a partir de una visión conectivista. Esto puede llevarse a cabo implementando sistemas de gestión de aprendizaje como ARMAGAeco-c (Mosquera \& Aguilar, 2015; Mosquera, Guevarra \& Aguilar, 2019), un middleware reflexivo basado en computación autonómica, cuya implementación proporciona el soporte computacional necesario para optimizar entornos de aprendizaje para el conocimiento conectivo. La implementación de este sistema puede llevarse a cabo de acuerdo con lo propuesto en (Mosquera \& Aguilar, 2016).

\section{Tabla 5}

Tareas de LA para el SaCI en relación a los procesos del eco-conectivismo

\begin{tabular}{cl}
\hline Tarea de LA & \multicolumn{1}{c}{ Actividad a incorporar en SaCI } \\
\hline $\begin{array}{c}\text { Agrupamiento y } \\
\text { enriquecimiento } \\
\text { semántico }\end{array}$ & $\begin{array}{l}\text { Incorporar actividades de aprendizaje que promuevan el trabajo colaborativo } \\
\text { utilizando herramientas sociales de desarrollo y reflexión. El objetivo de esto } \\
\text { es utilizar el aprendizaje basado en proyectos colaborativos, con el objetivo } \\
\text { de producir, espontáneamente, comunidades de aprendizaje. Esto permite que } \\
\text { los alumnos del SaCI incorporen recursos de aprendizaje en su PLE para } \\
\text { permitir la comunicación y, por ende, el establecimiento de la PKN de cada } \\
\text { estudiante. }\end{array}$ \\
\hline $\begin{array}{c}\text { Segmentación y } \\
\text { alineación }\end{array}$ & $\begin{array}{l}\text { Establecer el umbral de supervivencia ecológica. Esto permite la evolución } \\
\text { del proceso de aprendizaje en SaCI,hacia una ecología de conocimiento } \\
\text { "sana" donde se reconocen indicadores de conocimiento conectivo,. }\end{array}$ \\
\hline Recomendación & $\begin{array}{l}\text { Especificar los recursos de aprendizaje (repositorios y herramientas de social } \\
\text { media) que serán utilizados para la adecuación delos PLE y comunidades, } \\
\text { derivados de las migraciones de PLEs. }\end{array}$ \\
\hline
\end{tabular}

Fuente: Elaboración Propia

Volumen 23, $N^{\circ} 3$, Septiembre-Diciembre 2019. ISSN 2244-7296 


\section{ECO-CONECTIVISMO: MODELANDO EL CONOCIMIENTO CONECTIVO}

Diego Mosquera Uzcátegui y José Lisandro Aguilar

(pp.158-185)

La incorporación de las nuevas actividades en el SaCI y la integración de ARMAGAecoc al conjunto de ciclos autonómicos de tareas del SaCI, permitirá caracterizar ecologías de conocimiento estables desde la perspectiva conectivista. Esto último significa que el SaCI no solo promueve y genera conocimiento del tipo cualitativo y cuantitativo, (a partir de las teorías clásicas de aprendizaje), sino también explota las capacidades físicas y lógicas de su entorno para generar conocimiento conectivo.

\section{Conclusiones y Trabajos Futuros}

Las TIC han sido introducidas en el campo de la educación con la intención de extender y mejorar los procesos educativos. Sin embargo, las teorías clásicas de aprendizaje como las constructivistas, conductistas y cognitivistas, se han visto limitadas para dar respuesta a los modelos de aprendizaje que han emergido como consecuencia del uso de las TIC. Uno de los principales vacíos que tienen las teorías clásicas de aprendizaje es la imposibilidad de describir tipos de conocimiento que ocurren más allá de las fronteras de la percepción y razonamiento del individuo.

En este trabajo se introduce el uso del conectivismo desde su perspectiva epistemológica. Esto nos permite comprender cómo ocurre el conocimiento conectivo en entornos de aprendizajes socializados y mediados por las TIC. En particular, usamos el conectivismo, y el paradigma de ecologías de conocimiento, como base conceptual para describir el ecoconectivismo; un marco referencial para implementar entornos de aprendizaje que explotan el conocimiento conectivo.

El eco-conectivismo busca desarrollar la competencia de la praxis conectivista, dentro de un proceso de aprendizaje emergente, dirigido, fundamentalmente, por las condiciones de AIDA. Además, utiliza la noción de PLE y sus criterios diseño, para encontrar la articulación entre el aprendizaje formal y el aprendizaje informal.

En el eco-conectivismo se propone un modelo pedagógico empírico inspirado en conceptos provenientes de la ecología, cuyos organismos evolutivos son los PLE. En

Volumen 23, $N^{\circ} 3$, Septiembre-Diciembre 2019. ISSN 2244-7296 


\section{ECO-CONECTIVISMO: MODELANDO EL CONOCIMIENTO CONECTIVO}

Diego Mosquera Uzcátegui y José Lisandro Aguilar

(pp.158-185)

particular, el concepto de sucesión ecológica permite representar los momentos pedagógicos del aprendizaje en tres procesos evolutivos para los PLE: configuración, estabilización y unificación. Cada uno de estos procesos utiliza técnicas de analíticas de datos que permiten mejorar la gestión de los procesos de aprendizaje. En el caso del proceso de configuración eco-conectivista se utilizan técnicas descriptivas para comprender el estado actual de la ecología del conocimiento. El proceso de estabilización utiliza técnicas predictivas para estimar aquello que es desconocido del proceso de aprendizaje, Por último, el proceso de unificación eco-conectivista utiliza técnicas prescriptivas para que, de manera autonómica, el sistema ejecute las recomendaciones sobre las acciones que se han de seguir para optimizar la ecología de conocimiento.

Estos tres procesos eco-conectivistas se definen de manera abstracta, desde una perspectiva computacional, para ser implementados como un ciclo autonómico de tareas. En ese sentido, ARMAGAeco como la arquitectura computacional necesaria para proveer ambientes de aprendizaje eco-conectivistas, debe ser extendido con las tareas de LA y los ciclos autonómicos específicos para el aprendizaje conectivista.

Por otro lado, para el SaCI el eco-conectivismo proporciona una mejora en la gestión de los procesos de aprendizaje de acuerdo con dos aspectos fundamentales. Por un lado, provee nuevos indicadores de rendimiento en cuanto al desarrollo de capacidades metacognitivas ("saber dónde" y "saber transformar") del alumno, así como su integración y aportación en trabajos colaborativos. Por otro lado, integra un nuevo ciclo autonómico de tareas como un eje transversal para descubrir propiedades del ambiente, que son importantes en un aprendizaje del tipo "aprender-haciendo". En particular, el conocimiento conectivo proporciona información de AIDA, como propiedades deseables en un proceso de aprendizaje constructivista bajo el paradigma "aprender-haciendo".

En cuanto a los trabajos futuros creemos que un paso importante es la formalización del eco-conectivismo como un modelo teórico de sucesión ecológica para comunidades de aprendizaje. En específico, es importante describir el proceso eco-conectivista como un

Volumen 23, N³, Septiembre-Diciembre 2019. ISSN 2244-7296 


\section{ECO-CONECTIVISMO: MODELANDO EL CONOCIMIENTO CONECTIVO}

Diego Mosquera Uzcátegui y José Lisandro Aguilar

(pp.158-185)

modelo de Markov de sucesión ecológica. Las cadenas de Markov son procesos estocásticos, donde la ocurrencia de un evento depende solo de la ocurrencia del evento inmediato precedente, la cual estima los cambios entre estados ecológicos en forma de probabilidades. Entre las ventajas más significativas del uso de cadenas de Markov en el eco-conectivismo tenemos: incorporación de la disposición espacial en forma de estados de abundancias o riquezas específicas, identificación de los cambios en la dominancia numérica de individuos, facilita la cuantificación de las probabilidades de transición entre estados ecológicos (en lugar de funciones que describen procesos ecológicos específicos); entre otras.

Por otro lado, consideramos que la noción de diversidad introducida en este artículo (basada en el umbral de supervivencia ecológica) como una propiedad emergente de las ecologías de conocimiento, no es suficientemente robusta. Para mejorar esto, es necesario incorporar tres elementos al cálculo de la diversidad: 1) la riqueza individual de entidades de un ecosistema; 2) la tasa de cambio o reemplazo en la composición de entidades entre diferentes ecosistemas; y 3) la riqueza de entidades del conjunto de ecosistemas que integran la ecología del conocimiento. A esta forma de analizar la ecología del conocimiento se le conoce, respectivamente, como diversidad alfa, beta y gamma. Además, en la literatura, es posible encontrar métodos específicos para su medición, siendo los más utilizados los índices de similitud/disimilitud o distancia.

Finalmente, durante la investigación, pudimos observar que existe investigación relacionada en un área relativamente nueva, llamada aprendizaje invisible (un paradigma que se encuentra en plena fase de desarrollo). El aprendizaje invisible se centra en cómo aprender, y no qué aprender. Se basa en el uso de estrategias didácticas que incorporan la solución de problemas, la elaboración de proyectos, los laboratorios de experimentación, las incubadoras de ideas, las comunidades de aprendizaje, entro otras. El aprendizaje invisible puede verse como el conjunto de principios que definen el diseño instruccional del aprendizaje informal y su articulación con el aprendizaje formal. Bajo este enfoque se busca explorar un panorama de opciones para la creación de futuros relevantes para la educación

Volumen 23, N³, Septiembre-Diciembre 2019. ISSN 2244-7296 


\section{ECO-CONECTIVISMO: MODELANDO EL CONOCIMIENTO CONECTIVO}

Diego Mosquera Uzcátegui y José Lisandro Aguilar

(pp.158-185)

actual. En este sentido, creemos que gran parte de nuestra propuesta puede alinearse en el marco del desarrollo del aprendizaje invisible, lo que daría una nueva base conceptual a nuestros modelos de aprendizaje.

\section{Referencias}

Aguilar, J. (2014) Introducción a los Sistemas Emergentes, Talleres Gráficos, Universidad de Los Andes

Aguilar, J., Riofrio, G., Encalada, E., \& Guamán, D. (2015). Business Intelligence applied to Learning Analytics in student-centered learning processes. XLI Conferencia Latinoamericana en Informática , 567-576.

Aguilar, J., Valdivieso, P., Cordero, J., Riofrio, G., \& Encalada, E. (2015). A general framework for Learning Analytic in a Smart Classroom. Society for Learning Analytics Research (SOLAR) , 1-14.

Aguilar, J., Valdiviezo, P., Cordero, J., \& Sánchez, M. (2015). Conceptual Design of a Smart Classroom Based on Multiagent Systems. Int'l Conf. Artificial Intelligence - IICAI, 471-477.

Barabási, A.-L., \& Albert, R. (1999). Emergence of scaling in random networks. Science 286, 509-512.

Cobo, C., \& Moravec, J. (2011). Aprendizaje invisible. Hacia una nueva ecología de la educación. Barcelona: Universidad Nacional de Andalucia.

Dawnes, S. (2012). Connectivism and Connective Knowledge. Canada: National Research Council.

Downes, S. (2007). What connectivism is. (Último acceso, 13-06-2007). Obtenido de http://halfanhour.blogspot.com/2007/02/what-connectivism-is.html

Droege, P. (1997). Intelligent Environments - Spatial Aspect of the Information Revolution. Oxford: Elsevier.

Ferguson, R. (2012). Learning analytics: drivers, developments and challenges. International Journal of Technology Enhanced Learning. , 304-317.

Ferguson, R., \& Buckingham, S. (2012). Social Learning Analytics: Five Approaches. International Conference on Learning Analytics \& Knowledge .

García, I. (2009). Teoría de la conectividad como solución emergentes a las estrategias de aprendizaje innovadoras. Revista Electrónica de Humanidades, Educación y Comunicación Social (REDHECS) , 1-25.

Volumen 23, $N^{\circ} 3$, Septiembre-Diciembre 2019. ISSN 2244-7296 


\section{ECO-CONECTIVISMO: MODELANDO EL CONOCIMIENTO CONECTIVO}

Diego Mosquera Uzcátegui y José Lisandro Aguilar

(pp.158-185)

Ibañez, J. (2003). Acceso a la información y aprendizaje informal en Internet. Revista científica de comunicación y educación. , 31-38.

Mosquera, D., \& Aguilar, J. (2015). Middleware Reflexivo para la gestión de Aprendizajes Conectivistas en Ecologías de Conocimientos (eco-conectivismo). Latin-american Journal of Computing - LAJC , 25-32.

Mosquera, D., \& Aguilar, J. (2016). Modelo Autonómico Genérico para Middlewares de Gestión de Entornos de Aprendizaje Eco-conectivistas. Investigación inédita.

Olmo, F., Llera, J., \& Arias, M. (1999). El Aula Inteligente: una experiencia educativa innovadora. Revista española de pedagogía. , 83-109.

Pardo. (2014). Designing Learning Analytics Experiences, in Learning Analytics From Research to Practice. New York: Springer.

Sánchez, M., \& Campos, L. (2014). La mejora de los aprendizajes desde el "Aula Inteligente": Experiencia en la IE Coronel José Joaquín Inclán - Piura - Perú. Memorias del Congreso Iberoamericano de Ciencia, Tecnología, Innovación y Educación, 1-19.

Sánchez, M., Aguilar, J., Cordero, J., \& Valdiviezo, P. (2015). Basic features of a Reflective Middleware for Intelligent Learning Environment in the Cloud (IECL). Computer Aided System Engineering (APCASE) , 1-6.

Schunk, D. (2012). Teorías del aprendizaje. Una perspectiva educativa. Sexta edición. EEUU: Pearson.

Siemens, G. (2005). Connectivism: A learning theory for the digital age. International Journal of Instructional Technology \& Distance Learning .

Siemens, G. (2006). Knowing knowledge. Recuperado de http://www.knowingknowledge.com/

Volumen 23, $N^{\circ} 3$, Septiembre-Diciembre 2019. ISSN 2244-7296 